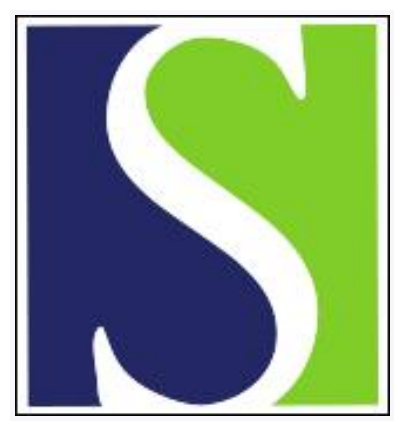

Scand J Work Environ Health 2002;28(5):289-292

https://doi.org/10.5271/sjweh.677

Issue date: Oct 2002

Are pushing and pulling strong risk factors for occupational shoulder disorders?

by Nelson NA

Affiliation: 2500 Brockman Blvd, Ann Arbor, MI 48104, United States.

Refers to the following texts of the Journal: 1999;25(5):387-403

1999;25 suppl 4:31-35 2002;28(5):293-303 2000;26(4):283-291

Key terms: editorial; occupational shoulder disorder; pulling; pushing; risk factor

This article in PubMed: www.ncbi.nlm.nih.gov/pubmed/12432981 


\section{Are pushing and pulling strong risk factors for occupational shoulder disorders?}

Over the past decade, tremendous methodological improvements have been made in the epidemiologic study of occupational musculoskeletal disorders. Reviewers have criticized past use of the crosssectional study design $(1,2)$, and dozens of recent studies have moved to the prospective examination of the upper extremities and back pain. Other study characteristics that are commonly criticized include poor participation rates, use of overly broad or subjective definitions for relevant workplace exposures and health outcomes, use of varying definitions from one study to the next so that results are difficult to compare, and failure to adjust for the effects of important covariates $(3,4)$. These study limitations seem to have been addressed less consistently than the change in study design, but improvements have been notable in exposure assessment, use of standardized methods for outcome definition, and adequate control of important covariates. Some aspects of methodology are at odds with one another-for example, achieving a good participation rate becomes perhaps more difficult in a prospective study than in a cross-sectional study. Riihimäki (5) has stated that ". . . ascertainment of disease outcome and exposure assessment are crucial challenges ... [p 35]".

In this issue of the Scandinavian Journal of Work Environment \& Health, the prospective study of Hoozemans et al (6) examines questionnaire results for back and shoulder complaints over a 1-year period among 459 persons working in several industries identified as having high exposure to pushing and pulling, namely, rail catering, nursing homes, flower auctions, postal distribution centers, and refuse collection. The authors addressed many of the aforementioned methodological concerns, with interesting results. While no association was found between pushing and pulling and back pain, fairly strong relationships were seen for shoulder complaints. This relatively less studied anatomic area seems to have few established occupational risk factors (1), the results therefore being particularly notable. As with all studies, some aspects of this investigation made results difficult to interpret. The strength of the prevalence ratios that were observed invites further examination of the methodology and findings of the study.

The study population was especially selected for its high exposure to pushing and pulling, potential risk factors that have not been addressed often for back and shoulder disorders in the literature. [A question that arises is why pushing and pulling were combined, since the two motions are biomechanically distinct. The authors have pointed out this aspect in another paper (7) but also stated that the two activities have a great deal of overlap. The combination thus seems justified and the rationale might have been mentioned.] For three of the five occupational groups studied, unexposed referents working in the same facilities were included. A strong feature of the study is its exposure assessment, which was carried out using both subjective (self-reported questionnaire) and objective means. Objective work exposure data were collected at the beginning of the study using the TRAC system, an approach in which two persons directly observe a sample of workers in target jobs while recording work posture and activity on hand-held computers. The system appears to be of practical use for a number of workplace environments; several publications have described its development (8). TRAC was used to quantify the following important physical risk factors: standing, sitting, kneeling, walking, pushing, pulling, lifting, and carrying. The work postures that were observed included trunk flexion and rotation and work with arms elevated. Although observational exposure assessment methods are necessarily limited by their time and expense requirements, nearly $30 \%$ of the exposed and reference groups were observed for part or all of 1 day at 
the beginning of the study. Prebaseline work-related exposures were not quantified, and this lack may have had some bearing on the observed results.

In this cohort there appeared to be some potential for misclassification according to exposure status, as discussed by the authors. On the average, study members had worked 10 or more years in their current jobs at the beginning of the follow-up. Previous exposure was not quantified and examined in the analyses. The authors suggested that a healthy worker effect was operating in this population, in which workers who sustained injuries in previously highly exposed jobs later dropped out or were transferred to less exposed jobs. The highest prevalence ratios for shoulder (and back) pain were observed in the medium exposure groups; the finding, which the authors explained, could relate to differential healthy worker effects in higher and lower exposure groups. The ". . . high exposure group may have become relatively healthy and less susceptible to the development of musculoskeletal complaints, caused by either selection at the start of employment or the dropout of workers susceptible to complaints in the course of early years of employment (35). The selection may have been less pronounced in the medium exposure group, which remained susceptible to complaints [6, p 313]". This difficulty is common in musculoskeletal studies (9) and seems plausible in this case, since the majority of the participants $(83 \%)$ had the opportunity to move from the exposed to the reference groups before the baseline data were collected (postal and refuse workers had no unexposed reference jobs).

A more puzzling aspect of the exposure assessment relates to lifting and carrying, fairly consistent and strong risk factors for back pain (10). The authors examined a comprehensive list of workplace physical and psychosocial factors along with other covariates and found no strong associations between back pain and lifting or carrying. The investigators stated that this finding may have resulted because the study group had low exposure to these activities. This assumption seems somewhat unlikely for at least the nursing home group, which, at $38 \%$, was the largest occupational subset of the population. More to the point, it represented $46 \%$ of the reference population. Nursing home aides are highly exposed to heavy lifting (11), and this degree of exposure may have been likely for both those exposed to pushing and pulling and the reference groups. The appendix of the report does not reflect this possibility, perhaps because only the frequency of lifting is shown, and not the magnitude. The preponderance of nursing home employees in the study population may have influenced the weak association that was observed between lifting and back disorders. Perhaps the addition of a weight limit (such as 50 kilograms) to the lifting variable would have helped identify those lifting heavy loads and produced different results.

Health outcomes were defined in a standard manner using the Dutch Musculoskeletal Questionnaire. Back complaints were self-reported by roughly $50 \%$ of the study group at the beginning of the study and in the follow-up; shoulder complaints were reported by $25 \%-50 \%$. Complaint rates were similarly high in the exposed and reference groups. The complaint definitions were broad, including aches, pains, and discomfort occurring during the past 12 months. High-frequency outcomes present several difficulties, one of which is analytical. The authors used a Cox proportional hazard regression to address this issue. In addition to the analytical difficulties is the implication of using an outcome definition that encompasses episodes of unknown frequency, duration, or intensity of pain. The background rates of musculoskeletal disorders are high for the general population; using an outcome definition that includes episodes of mild severity that come and go frequently may cause greater difficulty in distinguishing those with and those without work-related disorders. Methodologically, misclassification of this sort would bias prevalence ratios toward one and may have influenced the results for both health outcomes. In a case-referent study of shoulder disorders and postural stress, Punnett et al (12) created case definitions by selfreported pain and a physical examination in an attempt to identify the most serious cases. While this level of effort may not be feasible for a large cohort study, questionnaire responses could have been used to restrict the case definitions. This procedure would have reduced the prevalence of outcome 
measures, but in the case of back disorders in particular (and perhaps shoulder complaints as well) it is still not clear what an optimal definition might be.

The results of this paper show that work-related pushing and pulling are associated with shoulder complaints, with adjusted prevalence ratios of 3.95 and 3.33 for the observed frequency and duration of exposure, respectively (in the medium exposure categories). These ratios apply to those with no previous shoulder complaints at the beginning of the study. The presence of complaints at the beginning of the study was strongly associated with the follow-up outcome, and interactions were observed between pain reported at the beginning of the study and pushing and pulling exposures. Nevertheless, the results related to shoulder complaints seem compelling, particularly in light of all the potential limitations that would seem to have biased prevalence ratios to lower rather than higher levels (if at all). The relative importance of pushing and pulling in comparison with other work-related physical risk factors is somewhat unclear, however. Most of the posture and activity variables appeared to be of little importance in this data set, but the authors raise the possibility that the population had little exposure to some of these variables (eg, lifting and carrying). Of particular concern would be the significant collinearity between pushing and pulling and some other workplace exposure that was not adequately measured. The paper provides evidence that such collinearity did not occur for many recognized workplace risk factors.

As demonstrated by this study, the use of a prospective study design does not guarantee that temporal relationships between musculoskeletal disorders and work exposures can be discerned in the absence of a young and newly hired cohort. Hoogendoorn et al (13) have suggested that more complex design and analysis approaches may be in order for the study of musculoskeletal disorders. They state that prospective studies may benefit from the incorporation of repeated measures of exposure and that an additional advantage is that changes in outcome can also be measured on a periodic basis. Perhaps more development of exposure-response models would assist in the selection of design and analysis approaches-for example, hypothesizing the relative contributions of acute, cumulative, high, and low exposures. The development of hypotheses relating to the level of expected tissue damage might assist in creating more specific definitions for health outcomes $(1,2,4)$.

\section{References}

1. Van der Windt DAWM, Thomas E, Pope DP, De Winter AF, Macfarlane GJ, Bouter LM. Occupational risk factors for shoulder pain: a systematic review. Occup Environ Med 2000;57:433-42.

2. Bongers PM, Kremer AM, ter Laak J. Are psychosocial factors, risk factors for symptoms and signs of the shoulder, elbow, or hand/wrist?: A review of the epidemiological literature. Am J Ind Med 2002;41:315-42.

3. Hoogendoorn WE, van Poppel MNM, Bongers PM, Koes BW, Bouter LM. Physical load during work and leisure time as risk factors for back pain. Scand J Work Environ Health 1999;25:387-403.

4. National Research Council and Institute of Medicine, Panel on Musculoskeletal Disorders and the Workplace, Commis sion on Behavioral and Social Sciences and Education. Musculoskeletal disorders and the workplace: low back and upper extremities. Washington (DC): National Academy Press, 2001.

5. Riihimäki H. Musculoskeletal diseases - a continuing challenge for epidemiologic research. Scand J Work Environ Health 1999;25 suppl 4:31-5.

6. Hoozemans MJM, van der Beek AJ, Frings-Dresen MHW, Van der Woude LHV, Van Dijk FJH. Low-back and shoulder complaints among workers with pushing and pulling tasks. Scand J Work Environ Health 2002;28:307-317.

7. van der Beek AJ, Hoozemans MJM, Frings-Dresen MHW, Burdorf A. Assessment of exposure to pushing and pulling in epidemiological field studies: an overview of methods, exposure measures, and measurement strategies. Int J Ind Ergon 1999;24:417-29.

8. van der Beek AJ, van Gaalen LC, Frings-Dresen MHW. Working postures and activities of lorry drivers: a reliability study of on-site observation and recording on a pocket computer. Appl Ergon 1992;23:331-6.

9. Punnett L. Adjusting for the healthy worker selection effect in cross-sectional studies. Int J Epidemiol 1996;25:1068-76.

10. Bernard BP. Musculoskeletal disorders and workplace factors: a critical review of epidemiological evidence for workrelated musculoskeletal disorders of the neck, upper extremities, and low back. Cincinnati (OH): National Institute for Occupational Safety and Health, US Department of Health and Human Services, 1997. DHHS report, no 97-141.

11. Collins JW, Owen BD. NIOSH research initiatives to prevent back injuries to nursing assistants, aides, and orderlies in 
nursing homes. Am J Ind Health 1996;29:421-4.

12. Punnett L, Fine LJ, Keyserling WM, Herrin GD, Chaffin DB. Shoulder disorders and postural stress in automobile assembly work. Scand J Work Environ Health 2000;26:283-91.

13. Hoogendoorn WE, Bongers PM, de Vet HCW, Twisk JWR, Van Mechelen W, Bouter LM. Comparison of two different approaches for the analysis of data from a prospective cohort study: an application to work related risk factors for low back pain. Occup Environ Med 2002:59:459-65.

Nancy A Nelson, PhD

2500 Brockman Blvd

Ann Arbor, MI 48104

USA

[E-mail:nanphilco@aol.com] 